\title{
Eulersche Knicklast
}

\author{
Philippe Cachot, Markus Meier, Marcel Steiner und Urs Wüst
}

Philippe Cachot studierte Maschinenbau an der Fachhochschule beider Basel. Seit 2008 ist er als Berechnungsingenieur bei der Firma Helbling Technik AG tätig.

Markus Meier promovierte im Fach Physik an der ETH Zürich. Seit 2005 ist er als Lehrer für Mathematik und Physik an der Alten Kantonsschule Aarau tätig.

Marcel Steiner promovierte im Fach Mathematik an der EPF Lausanne. Seit 2002 ist er Professor für Mathematik an der Fachhochschule Nordwestschweiz.

Urs Wüst studierte Maschinenbau an der Ingenieurschule beider Basel. Nach seinem Studium und einigen Jahren als Entwicklungsingenieur in Forschung und Industrie, war er an der Fachhochschule beider Basel als wissenschaftlicher Mitarbeiter tätig.

\section{Historischer Abriss zur Knickformel}

Die Knickformel, die von Euler erstmals 1744 formuliert wurde, ist das Resultat einer langen Suche nach einer Balkentheorie.

Die Elastizitätstheorie entwickelte sich über einen langen Zeitraum. Sie nahm ihren Anfang bei Galilei im 17. Jahrhundert und wurde im 19. Jahrhundert von Navier und Cauchy vollendet. Die Vorstellungen und Begriffe der heutigen Elastizitätstheorie Spannung, Elastizitätsmodul, Biegelinie, neutrale Faser, Schnittlasten, Biegemomente, Flächenmomente usw. - entwickelten sich schrittweise und wurden über einen langen Zeitraum eingeführt (vgl. [9], [10] und [11]).

Die Elastizitätstheorie nahm ihren Anfang mit einer Theorie von Galilei (1564-1642) 1638. Seine Fragestellung beschränkte sich auf das Reissen oder Brechen eines Trag-

Das klassische Problem der Auffindung der Knicklast zylindrischer Stäbe unter Last wurde bereits von Euler untersucht. Seine berühmte Knickformel gehört heute zum Standardrepertoire der Ingenieurausbildung. Nach einem historischen Abriss zur Knickformel leiten die Autoren die Differentialgleichung für einen beidseitig gelenkig gelagerten Stab unter axialer Einwirkung einer Last her. Daraus wird für verschiedene Stabgeometrien die Formel für die Eulersche Knicklast gewonnen. Aus der numerischen Lösung für einen stückweise konischen Stabe wird sodann auf die technisch optimale Form des Stabes geschlossen, bei der die Knicklast maximiert wird. 


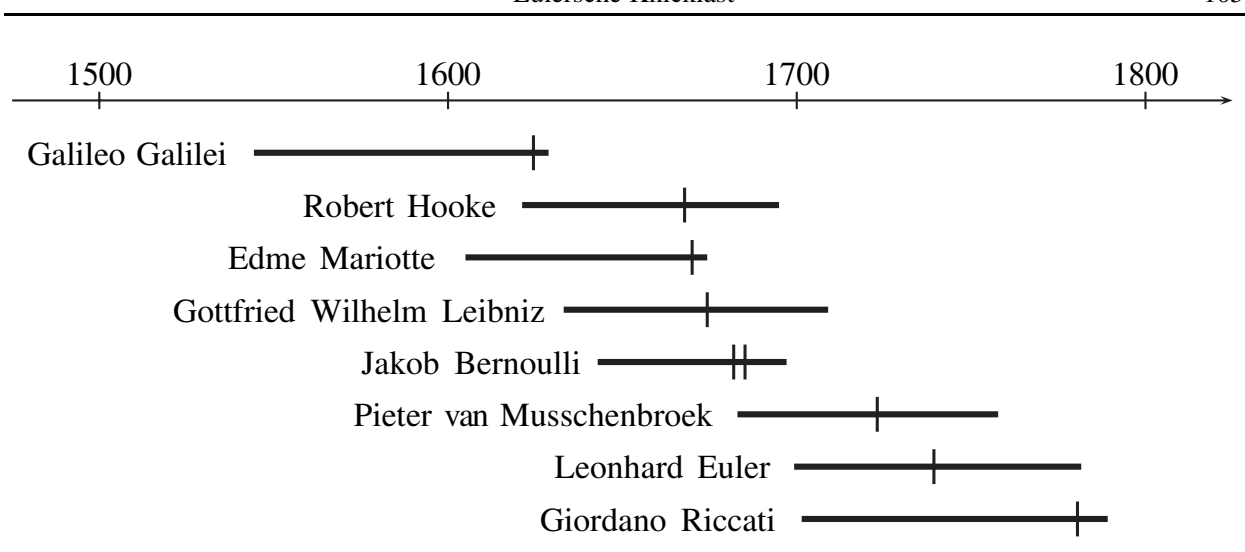

werkes. In seinen Discorsi betrachtete er einen einseitig eingespannten horizontalen Balken, der am anderen Ende durch ein Gewicht belastet wurde. Galilei nahm an, dass alle Längsfasern die gleiche Zugspannung aufnehmen, und dass sich der Balken um die untere Kante der Einspannung drehen würde. Wenn auch die Annahme der über den Balkenquerschnitt konstanten Zugspannung zu einer quantitativ falschen Formel führte, konnte Galilei doch Aussagen über das Verhalten von Balken mit verschiedenen Abmessungen machen.

Einen wichtigen Beitrag zur Begriffsbildung leistete Robert Hooke (1635-1703) 1678 mit seinem nach ihm benannten Federgesetz. Er beschrieb die Auslenkung eines Drahtes unter Zug, einer Spiralfeder unter einem Drehmoment oder die Biegung eines Balkens unter Last als proportional, also linear, zur angreifenden Kraft. Allerdings verwendete Hooke noch nicht Begriffe wie Spannung oder Elastizitätsmodul. Er unterschied aber zwischen Druck-, Zug- und Biegebeanspruchung, wobei er feststellte, dass die Fasern teils verlängert, teils verkürzt werden. Indirekt fand er so die neutrale Faser.

Die Ideen von Galilei wurden von Edme Mariotte (1620-1684) 1680 und Gottfried Wilhelm Leibniz (1646-1716) 1684 unter Berücksichtigung von elastischen Fasern weitergeführt, ohne aber auf das kurz zuvor von Hooke formulierte Gesetz Bezug zu nehmen. Beide nahmen an, dass die Zugkraft über den Balken linear variieren würde. Obwohl sie verbesserte Ansätze wählten, waren die Resultate falsch. Mariotte stellte Versuche mit Glas und Holzstäben an und stellte Unstimmigkeiten mit den Formeln von Galilei fest.

Bisher wurden bei den Überlegungen für eine Bruchtheorie nur die Kräfte bei der Balkenbiegung betrachtet, nicht aber die Form der Biegelinie. Jakob Bernoulli (1655-1705) ging einen wesentlichen Schritt weiter, indem er versuchte, die Biegelinie - auch elastische Linie oder Elastika genannt - eines elastischen Bandes zu berechnen. Er stellte dieses Problem 1691, teilte gleichzeitig seine Lösung als Anagramm mit und veröffentlichte 1694, nachdem keine Lösungen eingegangen waren, die korrekte Lösung für die Biegekurve. Bernoulli konnte hier erstmals gekonnt das kraftvolle Instrument der Differentialrechnung einsetzen. Seine Beschreibung der Biegelinie erfolgt mit einer geometrischen Konstruktion, die einen geübten Leser auch heute stark fordert. Bernoulli lieferte keinen Beweis für seine Konstruktion. 
Pieter van Musschenbroek (1682-1761) veröffentlichte 1729 Resultate von systematischen Versuchen zum Knick- resp. Bruchverhalten von Holzstäben unter einer Drucklast. Er fand die Knicklast $F$ proportional zu

$$
F \propto \frac{d^{2} b}{l^{2}} .
$$

Hier bedeutet $b$ die Breite und $d$ die Dicke des Stabes. Diese Beziehung gibt die Abhängigkeit von der Länge korrekt wieder, falsch ist hingegen der Einfluss der Querabmessungen des Stabes.

Nach Jakob Bernoulli befasste sich Leonhard Euler (1707-1783) mit der Bestimmung der elastischen Linie eines gebogenen Balkens. Im Zusammenhang mit seinem Meisterstück, der Variationsrechnung, formulierte er das Problem wie folgt:

Unter allen Kurven derselben Länge, die durch zwei Punkte gehen und in diesen Punkten von der Lage nach gegebenen Geraden tangiert werden, ist diejenige zu finden, für welche der Wert des Ausdrucks $\int \frac{1}{R^{2}} \mathrm{~d}$ s ein Minimum wird.

Hier bedeutet $\mathrm{d} s$ das Bogenelement entlang der Kurve und $R$ den Krümmungsradius der elastischen Linie. Euler behandelte zahlreiche Fälle, darunter denjenigen, der ihn $1744 \mathrm{zu}$ seiner berühmten Knickformel führte: Er betrachtete eine Säule, die anders als bei den bisherigen Überlegungen nur von einer Kraft $F$ in Achsenrichtung belastet wurde. Er stellte fest, dass eine Verbiegung, also ein Ausknicken, nur möglich war, wenn die Kraft $F$ eine kritische Grenze überschritt:

$$
F>\frac{\pi^{2}}{l^{2}} \tilde{E} k^{2}
$$

Hier bedeutet $l$ die Länge des Stabes und $\tilde{E} k^{2}$ eine von der Elastizität und den Abmessungen der Säule abhängige Konstante. Vor Euler wurde nur der Fall betrachtet, bei dem die Kraft quer zum Balken wirkte, die Biegung des Balkens ist damit stetig und proportional zur einwirkenden Kraft. Euler hat für diesen neuen Fall erstmals ein Stabilitätsproblem erkannt und mathematisch behandelt. Er zeigte, dass das Knickverhalten nur von den elastischen Eigenschaften und der Form abhängt, nicht aber von den Brucheigenschaften des verwendeten Materials.

Die Grösse $\tilde{E} k^{2}$ in der Knickformel von Euler verlangt noch nach einer Erklärung. Euler bemerkte dazu, dass $\tilde{E} k^{2}$ erstens von der Natur des Materials abhängt, zweitens der Breite des Bandes, und drittens zur zweiten Potenz der Dicke des Bandes proportional ist. Die von Euler verwendete Grösse $\tilde{E}$ ist nicht der heute verwendete Elastizitätsmodul (vgl. Kap. 2). Die Grösse $\tilde{E} k^{2}$ wird von Euler als Moment du Ressort bezeichnet. Euler hat sich hinsichtlich des Einflusses der Querschnittsabmessungen geirrt. Diesen Fehler hat bereits Giordano Riccatti (1709-1790) 1782 bemerkt. Euler verwendete in seiner Behandlung einen phänomenologischen Ausdruck für die Elastizität, ohne genauer auf die involvierten Grössen einzugehen. Dies erstaunt, denn Euler hatte sich bereits 1727 in Basel mit den elastischen Schwingungen eines Stabes beschäftigt. In dieser Arbeit verwendete er eine Grösse, die dem Elastizitätsmodul entspricht, ohne ihr aber einen Namen zu geben. 


\section{Analytische und davon abgeleitete numerische Lösung}

Ein schlanker Stab der Länge $l$ werde in Richtung seiner Achse durch die Kraft $F$ belastet. Die Enden seien je nach Situation verschiedenartig gelagert oder eingemauert. Wir betrachten nur den Fall, bei dem beide Enden beweglich gelagert sind und der Stab nach allen Seiten ausbrechen kann (vgl. Abb. 1). Wie gross ist die Kraft $F$, bei der der Stab ausknickt?

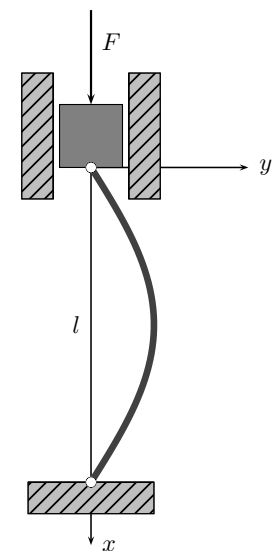

Abb. 1 Ein schlanker Stab, der in Richtung seiner Achse durch die Kraft $F$ belastet wird. Beide Enden sind beweglich gelagert, d.h. $y(0)=y(l)=0$.

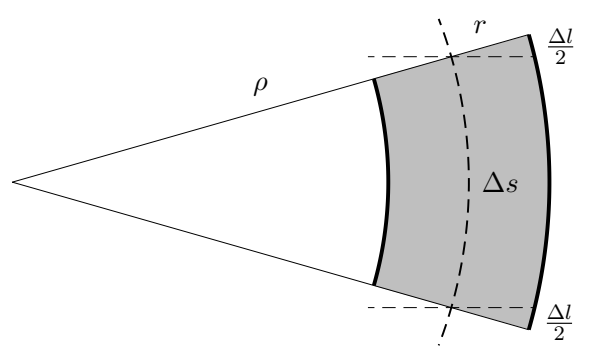

Abb. 2 Durchbiegung eines Stabes mit Durchmesser $2 r$. Der Krümmungsradius der Mittellinie sei $\rho$ und die Verlängerung der äusseren Fasern sei $\Delta l$.

Wird ein Stab durch ein Moment $M$ auf Biegung beansprucht, so wirken auf der einen Seite Zugspannungen verbunden mit einer Verlängerung der Fasern und auf der anderen Seite Druckspannungen verbunden mit einer Verkürzung der Fasern. In der Mitte befindet sich dann eine Schicht, die ihre Länge beibehält. Das Verhältnis des Krümmungsradius $\rho$ zur Länge der Mittellinie $\Delta s$ ist gleich dem Verhältnis des Radius des Stabes $r$ zur Längenänderung $\Delta l$, d.h.

$$
\frac{\rho}{\Delta s}=\frac{r}{\Delta l}
$$

(vgl. Abb. 2). Das Hookesche Gesetz, [6], besagt weiter, dass

$$
\frac{\Delta l}{\Delta s}=\frac{\sigma}{E},
$$

wobei $\sigma$ die Biegespannung und $E$ den Elastizitätsmodul beschreibt. Die Biegespannung lässt sich mit Hilfe des wirkenden Momentes $M$ und des axialen Flächenträgheitsmomentes $I$ des Stabquerschnitts beschreiben:

$$
\sigma=\frac{M}{I} r .
$$

Aus den Gl. (1) bis (3) folgt unmittelbar, dass für die Krümmung $\frac{1}{\rho}=\frac{M}{E T}$ gilt. Es sei $y$ die Auslenkung des Stabes an der Stelle $x, 0 \leq x \leq l$. Dann ist das Biegemoment

$$
M(y)=F y
$$


im betrachteten Koordinatensystem positiv und somit $y^{\prime \prime}<0$. Mit Hilfe der bekannten Formel für die Krümmung einer differenzierbaren Kurve erhalten wir die Differentialgleichung der elastischen Linie:

$$
\frac{y^{\prime \prime}}{{\sqrt{1+y^{\prime 2}}}^{3}}+\frac{F}{E I} y=0 .
$$

Uns interessieren nur kleine Auslenkungen im Vergleich zur Stablänge, damit ist der Tangenswert des Auslenkwinkels $y^{\prime}$ klein im Vergleich zu 1, und Gl. (5) vereinfacht sich zur bekannten linearen Differentialgleichung der elastischen Linie:

$$
y^{\prime \prime}+\frac{F}{E I} y=0 .
$$

Im Folgenden untersuchen wir kreisrunde Stäbe mit verschiedenen Querschnitten (vgl.

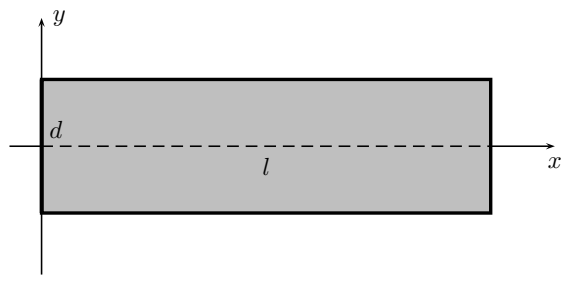

Abb. 3 Zylindrischer runder Stab mit Durchmesser $d$.

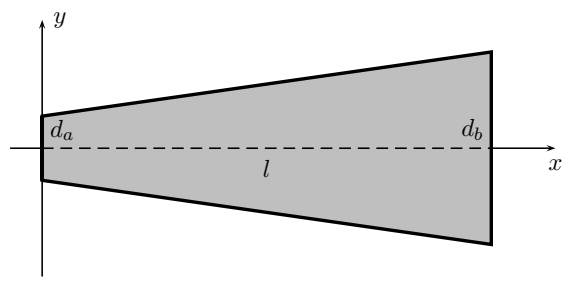

Abb. 4 Konischer runder Stab mit Konizität $k=\frac{d_{b}-d_{a}}{l}$.

Abb. 3 und 4), die auf beiden Seiten gelenkig eingebaut sind (vgl. Abb. 1), d.h., wir suchen Lösungen der Differentialgleichung (6) unter den Randbedingungen $y(0)=0$ und $y(l)=0$. Genauer interessieren wir uns eigentlich nur für den kleinsten nichttrivialen Eigenwert dieser Differentialgleichung.

Der zylindrische Stab. Zum Aufwärmen wollen wir die Lösung für den runden zylindrischen Stab (vgl. Abb. 3) repetieren. Das axiale Flächenträgheitsmoment des Stabquerschnittes ist $I=\frac{\pi}{64} d^{4}$, die Differentialgleichung (6) wird zu

$$
y^{\prime \prime}+\frac{\lambda^{2}}{d^{4}} y=0 \quad \text { mit } \quad \lambda^{2}=\frac{64 F}{\pi E}
$$

und hat die allgemeine Lösung

$$
y(x)=A \sin \left(\frac{\lambda}{d^{2}} x\right)+B \cos \left(\frac{\lambda}{d^{2}} x\right) .
$$

Aus der Randbedingung $y(0)=0$ folgt $B=0$; und aus $y(l)=0$ folgt $\frac{\lambda}{d^{2}} l=n \pi$, wobei $n \in\{0,1,2, \ldots\}$. Es gibt also für einen zylindrischen Stab nur für bestimmte diskrete Werte $\lambda$, resp. Kräfte $F$, nichttriviale Lösungen. Die kleinste solche Kraft

$$
F_{\text {Knicklast }}^{\text {zyl }}=\frac{\pi^{2}}{l^{2}} E I=\frac{\pi^{3}}{64} \frac{d^{4}}{l^{2}} E,
$$

bei der der Stab knickt, ergibt sich für $n=1$ und wird Eulersche Knicklast genannt. 
Der konische Stab. Wir betrachten einen runden konischen Stab der Länge $l$ mit dem linear zunehmenden Durchmesser $d(x)=d_{a}+k x$, wobei $k=\frac{d_{b}-d_{a}}{l}$ die Konizität ist (vgl. Abb. 4). Das axiale Flächenträgheitsmoment des Stabquerschnittes ist demzufolge $I(x)=\frac{\pi}{64} d(x)^{4}$. Damit wird die zu lösende Differentialgleichung (6) zu

$$
y^{\prime \prime}+\frac{\lambda^{2}}{\left(d_{a}+k x\right)^{4}} y=0 \text { mit } \quad \lambda^{2}=\frac{64 F}{\pi E} .
$$

Erstaunlicherweise lässt sich die allgemeine Lösung explizit angeben. Sie lautet

$$
y(x)=A \cdot\left(d_{a}+k x\right) \sin \left(\frac{\lambda}{k\left(d_{a}+k x\right)}\right)+B \cdot\left(d_{a}+k x\right) \cos \left(\frac{\lambda}{k\left(d_{a}+k x\right)}\right) .
$$

Die Lösung lässt sich auch als Sinusfunktion mit Phasenverschiebung darstellen:

$$
y(x)=R \cdot\left(d_{a}+k x\right) \sin \left(\frac{\lambda}{k\left(d_{a}+k x\right)}+\phi\right),
$$

wobei $R=\sqrt{A^{2}+B^{2}}$ und $\phi=\arctan \left(\frac{B}{A}\right)$ aus den Randbedingungen bestimmt werden. Aus $y(0)=0$ und $y(l)=0$ folgt $\frac{\lambda}{k d_{a}}+\phi=m \pi$ und $\frac{\lambda}{k d_{b}}+\phi=n \pi$, wobei $m, n \in\{0,1,2, \ldots\}$. Wir erhalten $\lambda=(m-n) \pi \frac{d_{a} d_{b}}{l}$, und somit gibt es auch für einen konischen Stab nur für bestimmte diskrete Werte $\lambda$, resp. Kräfte $F$, nichttriviale Lösungen. Die kleinste solche Kraft ( $m=1$ und $n=0)$ ist wiederum die Knicklast

$$
F_{\text {Knicklast }}^{\text {kon }}=\frac{\pi^{3}}{64} \frac{d_{a}^{2} d_{b}^{2}}{l^{2}} E \text {. }
$$

Für $d_{a}=d_{b}$ ergibt sich die Eulersche Knicklast des zylindrischen Stabes.

Der stückweise konische Stab. Da der konische Stab explizit berechenbar ist, untersuchen wir nun einen runden Stab der Länge $l$, der aus $N$ Konussen der Längen $l_{i}$ und der Konizitäten $k_{i}=\frac{d_{i+1}-d_{i}}{l_{i}}$ stetig zusammengesetzt ist (vgl. Abb. 5).

Auf jedem Intervall $\left[x_{i}, x_{i+1}\right]$ mit $x_{i}=\sum_{j=1}^{i-1} l_{j}$ und $i \in\{1, \ldots, N\}$ können wir die Lösung explizit angeben. Gl. (7) und (8) liefern

$y_{i}(x)= \begin{cases}A_{i} \sin \left(\frac{\lambda}{d_{i}^{2}}\left(x-x_{i}\right)\right)+B_{i} \cos \left(\frac{\lambda}{d_{i}^{2}}\left(x-x_{i}\right)\right), & k_{i}=0 \\ \left(d_{i}+k_{i}\left(x-x_{i}\right)\right)\left(A_{i} \sin \left(\frac{\lambda}{k_{i}\left(d_{i}+k_{i}\left(x-x_{i}\right)\right)}\right)+B_{i} \cos \left(\frac{\lambda}{k_{i}\left(d_{i}+k_{i}\left(x-x_{i}\right)\right)}\right)\right), & k_{i} \neq 0 .\end{cases}$

Die globale Lösung erhalten wir durch stetiges und differenzierbares Zusammenkleben der $N$ Lösungen $y_{1}, y_{2}, \ldots, y_{N}$ an den Schnittstellen $x_{2}, x_{3}, \ldots, x_{N}$ im Innern des Stabes. Wir erhalten ein lineares homogenes Gleichungssystem für $2 N$ Integrationskonstanten $A_{1}, B_{1}, \ldots, A_{N}, B_{N}$ mit der Koeffizientenmatrix $K$. Aus der Forderung einer stetigen Lösungskurve ergeben sich $N-1$ Gleichungen $y_{i}\left(x_{i+1}\right)=y_{i+1}\left(x_{i+1}\right)$ für 


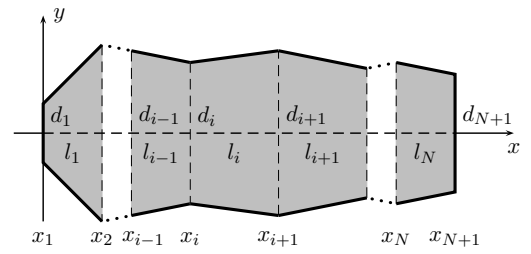

Abb. 5 Stückweise konischer Stab.

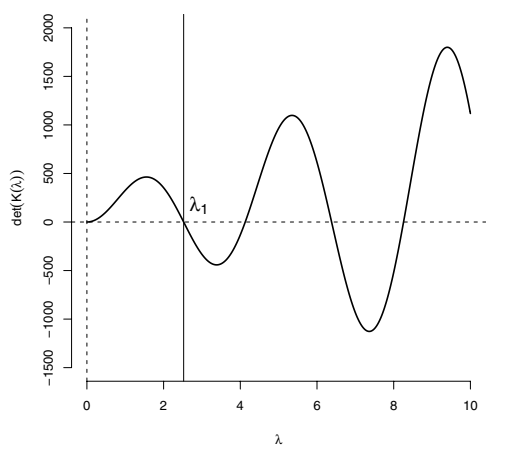

Abb. $6 \operatorname{det}(K(\lambda))$ des doppelkonischen Stabes mit $l_{1}=l_{2}=225 \mathrm{~mm}, d_{1}=d_{3}=13.19 \mathrm{~mm}$ und $d_{2}=22.41 \mathrm{~mm}$.

$i \in\{1, \ldots, N-1\}$, d.h. die Koeffizienten

$$
\begin{aligned}
K_{2 i, 2 i-1}(\lambda) & = \begin{cases}\sin \left(\frac{\lambda}{d_{i}^{2}} l_{i}\right), & k_{i}=0 \\
d_{i+1} \sin \left(\frac{\lambda}{k_{i} d_{i+1}}\right), & k_{i} \neq 0\end{cases} \\
K_{2 i, 2 i}(\lambda) & = \begin{cases}\cos \left(\frac{\lambda}{d_{i}^{2}} l_{i}\right), & k_{i}=0 \\
d_{i+1} \cos \left(\frac{\lambda}{k_{i} d_{i+1}}\right), & k_{i} \neq 0\end{cases}
\end{aligned}
$$

und

$$
\begin{aligned}
& K_{2 i, 2 i+1}(\lambda)= \begin{cases}0, & k_{i}=0 \\
-d_{i+1} \sin \left(\frac{\lambda}{k_{i+1} d_{i+1}}\right), & k_{i} \neq 0\end{cases} \\
& K_{2 i, 2 i+2}(\lambda)= \begin{cases}-1, & k_{i}=0 \\
-d_{i+1} \cos \left(\frac{\lambda}{k_{i+1} d_{i+1}}\right), & k_{i} \neq 0\end{cases}
\end{aligned}
$$

im Gleichungssystem. Aus der Differenzierbarkeit ergeben sich zusätzlich $N-1$ Gleichungen $y_{i}^{\prime}\left(x_{i+1}\right)=y_{i+1}^{\prime}\left(x_{i+1}\right)$ für $i \in\{1, \ldots, N-1\}$, d.h. die Koeffizienten

$$
\begin{aligned}
K_{2 i+1,2 i-1}(\lambda) & = \begin{cases}\frac{\lambda}{d_{i}^{2}} \cos \left(\frac{\lambda}{d_{i}^{2}} l_{i}\right), & k_{i}=0 \\
k_{i} \sin \left(\frac{\lambda}{k_{i} d_{i+1}}\right)-\frac{\lambda}{d_{i+1}} \cos \left(\frac{\lambda}{k_{i} d_{i+1}}\right), & k_{i} \neq 0\end{cases} \\
K_{2 i+1,2 i}(\lambda) & = \begin{cases}-\frac{\lambda}{d_{i}^{2}} \sin \left(\frac{\lambda}{d_{i}^{2}} l_{i}\right), & k_{i}=0 \\
k_{i} \cos \left(\frac{\lambda}{k_{i} d_{i+1}}\right)+\frac{\lambda}{d_{i+1}} \sin \left(\frac{\lambda}{k_{i} d_{i+1}}\right), & k_{i} \neq 0\end{cases}
\end{aligned}
$$




$$
\begin{aligned}
& K_{2 i+1,2 i+1}(\lambda)= \begin{cases}-\frac{\lambda}{d_{i}^{2}}, & k_{i}=0 \\
-k_{i+1} \sin \left(\frac{\lambda}{k_{i+1} d_{i+1}}\right)+\frac{\lambda}{d_{i+1}} \cos \left(\frac{\lambda}{k_{i+1} d_{i+1}}\right), & k_{i} \neq 0\end{cases} \\
& K_{2 i+1,2 i+2}(\lambda)= \begin{cases}0, & k_{i}=0 \\
-k_{i+1} \cos \left(\frac{\lambda}{k_{i+1} d_{i+1}}\right)-\frac{\lambda}{d_{i+1}} \sin \left(\frac{\lambda}{k_{i+1} d_{i+1}}\right), & k_{i} \neq 0\end{cases}
\end{aligned}
$$

im Gleichungssystem. Die Randbedingung links $y_{1}\left(x_{1}\right)=0$ definiert die Koeffizienten

$$
\begin{aligned}
& K_{11}(\lambda)= \begin{cases}0, & k_{i}=0 \\
d_{1} \sin \left(\frac{\lambda}{k_{1} d_{1}}\right), & k_{i} \neq 0\end{cases} \\
& K_{12}(\lambda)= \begin{cases}1, & k_{i}=0 \\
d_{1} \cos \left(\frac{\lambda}{k_{1} d_{1}}\right), & k_{i} \neq 0\end{cases}
\end{aligned}
$$

und die Randbedingung rechts $y_{N}\left(x_{N+1}\right)=0$ definiert die Koeffizienten

$$
\begin{array}{r}
K_{2 N, 2 N-1}(\lambda)= \begin{cases}\sin \left(\frac{\lambda}{d_{N}^{2}} l_{N}\right), & k_{N}=0 \\
d_{N+1} \sin \left(\frac{\lambda}{k_{N} d_{N+1}}\right), & k_{N} \neq 0\end{cases} \\
K_{2 N, 2 N}(\lambda)= \begin{cases}\cos \left(\frac{\lambda}{d_{N}^{2}} l_{N}\right), & k_{N}=0 \\
d_{N+1} \cos \left(\frac{\lambda}{k_{N} d_{N+1}}\right), & k_{N} \neq 0\end{cases}
\end{array}
$$

im Gleichungssystem. Alle anderen Koeffizienten sind null. Das homogene Gleichungssystem lässt nur nichttriviale Lösungen zu, wenn

$$
\operatorname{det}(K(\lambda))=0 .
$$

Dies ist nur für bestimmte diskrete Werte von $\lambda$ möglich (vgl. Abb. 6). Wiederum suchen wir, (diesmal) numerisch, den kleinsten nichttrivialen Wert $\lambda_{1}$ für den die Matrix $K(\lambda)$ singulär wird. Wir erhalten

$$
F_{\text {Knicklast }}=\frac{\pi}{64} \lambda_{1}^{2} E .
$$

Mit dieser Methode sind wir in der Lage, die Knicklast eines glatten Stabes approximativ zu berechnen, da jede Form beliebig genau durch einen stückweise konischen Stab angenähert werden kann. Weil die Koeffizientenmatrix $K$ Bandstruktur aufweist, eignet sich dieses Verfahren auch für relativ grosse $N$.

Der optimale Stab. Es stellt sich natürlich unmittelbar die Frage nach der Form des optimalen runden Stabes. Das heisst, wenn der Stab die Länge $l$ und das Volumen $V$ haben soll, welche Stabkontur maximiert die Knicklast? Mit unseren Vorarbeiten ist dieses Problem innerhalb der Familie der runden, stückweise konischen Stäbe mit numerischen Methoden lösbar. K.Y. Maalawi, [5], untersucht und optimiert mit ähnlichen Methoden stückweise zylindrische Stäbe.

Aus Symmetriegründen muss die Stabkontur symmetrisch zur Mitte der Stablänge verlaufen. Die Idee besteht in einer numerischen Maximierung des kleinsten nichttrivialen 
Wertes $\lambda_{1}$ bei Variation der Durchmesser $d_{1}, d_{2}, \ldots, d_{N+1}$ des stückweise konischen Stabes unter der Nebenbedingung der Konstanz des Stabvolumens

$$
V=\frac{\pi}{12} \sum_{j=1}^{N} l_{j}\left(d_{j}^{2}+d_{j} d_{j+1}+d_{j+1}^{2}\right) .
$$

Diese Optimierung kann mit Hilfe geeigneter Computeralgebrasoftware durchgeführt werden. In unserem Fall wurde die freie Software R, [8], verwendet. Dabei übernahm die Funktion opt im mit method="Nelder - Mead" (vgl. [7]) die Optimierung. Mit einem symmetrischen doppelkonischen Stab kann die Optimierung gestartet werden.

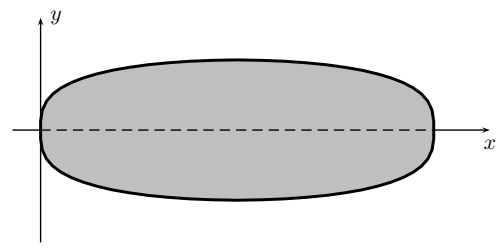

Abb. 7 Mathematisch optimaler Stab. An den beiden Enden des Stabes wird der Radius null Somit ist dieser Stab technisch nicht sinnvoll.

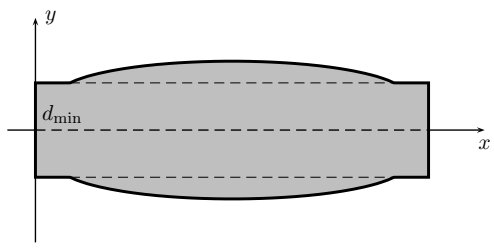

Abb. 8 Technisch optimaler Stab unter den Nebenbedingungen $d_{i} \geq d_{\min }$ für alle $i \in$ $\{1, \ldots, N+1\}$ und für einen vorgegebenen minimalen Durchmesser $d_{\text {min }}$.

Die optimale Form (vgl. Abb. 7) überrascht uns wenig, denn schon die antiken Säulen sind bauchig. Leider ist diese mathematisch optimale Lösung technisch nicht sinnvoll, da der Durchmesser an den beiden Enden gegen null geht. Dies hätte bei einer technischen Realisierung zur Folge, dass die Druckspannung $\sigma_{\text {Druck }}$ an diesen Stellen des Stabes die Streckgrenze $\sigma_{0.2}$ des Werkstoffes überschreitet. Hierbei träte Quetschen im Bereich der Stirnseiten ein. Dadurch würde der Werkstoff zu fliessen beginnen und plastische Deformation wäre die Folge davon. Um dies zu verhindern, müssen wir für den technisch optimalen Knickstab einen Mindestdurchmesser $d_{\min }$ für die Stabenden definieren. Dieser resultiert aus der Bedingung, dass die Druckspannung $\sigma_{\text {Druck }}$ unter Berücksichtigung eines Sicherheitsfaktors von $S=1.5$ die Streckgrenze $\sigma_{0.2}$ des verwendeten Stabwerkstoffes nicht überschreiten darf, d.h.

$$
\sigma_{\text {Druck }}=\frac{F_{\text {Knicklast }}}{A}=\frac{4 F_{\text {Knicklast }}}{\pi d_{\min }^{2}} \leq \frac{\sigma_{0.2}}{S} .
$$

Es folgt

$$
d_{\text {min }}=\sqrt{\frac{4 S F_{\text {Knicklast }}}{\pi \sigma_{0.2}}} .
$$

Somit sind wir gezwungen, die Optimierung unter den zusätzlichen Nebenbedingungen $d_{i} \geq d_{\min }$ für alle $i \in\{1, \ldots, N+1\}$ durchzuführen (vgl. Abb. 8).

Die Frage, ob die mathematisch optimale Form durch eine explizite Funktion beschrieben werden kann, beantwortet J.B. Keller, [4]. Er leitet die parametrische Form

$$
c(\vartheta)=\left(\frac{l}{\pi}\left(\vartheta-\frac{1}{2} \sin (2 \vartheta)\right), \sqrt{\frac{4}{3 \pi} \frac{V}{l}} \sin (\vartheta)\right) \text { mit } \vartheta \in[0, \pi]
$$


für die obere Hälfte der mathematisch optimalen Stabkontur explizit her. Damit erhält er den ersten nichttrivialen Eigenwert

$$
\lambda_{1}=\frac{8}{\sqrt{3}} \frac{V}{l^{2}}
$$

des Stabes. Die daraus berechnete Knicklast $F_{\text {Knicklast }}^{\text {math.opt }}=\frac{\pi}{3} \frac{V^{2}}{l^{4}} E$ gibt uns ein absolutes Maximum, das aber technisch nicht realisierbar ist. Unsere numerisch gefundene Lösung (vgl. Abb. 7) stimmt innerhalb der Rechengenauigkeit mit der exakten Lösung ausgezeichnet überein.

In der Literatur finden sich zahlreiche weitere numerische Untersuchungen mit Hilfe der Methode der finiten Elemente (vgl. [3] und die Referenzen darin). Wird zusätzlich die Forderung eines kreisrunden Stabquerschnittes zugunsten eines konvexen Querschnittes abgeschwächt, dann ist ein Querschnitt mit einem gleichseitigen Dreieck optimal (vgl. [4]).

\section{Finite Elemente Lösung}

Finite Elemente Simulationen (FEM) werden in der Industrie zur Untersuchung von kritischen Bauteilen und Baugruppen eingesetzt. Je nach Medium sind unterschiedliche Berechnungsmethoden erforderlich. In den Gebieten der Strukturmechanik, der Strömungsmechanik oder bei thermischen Untersuchungen sind diese Methoden weit verbreitet. Bei Simulationen mit finiten Elementen sind zwei Grundsätze zu beachten. Das mathematische Modell ist so zu wählen, dass die erwünschte Antwort innerhalb der geforderten Rechengenauigkeit liegt und diese in möglichst kurzer Zeit mit minimalem Kostenaufwand erreicht wird. Die Einflussnahme erfolgt im Wesentlichen über die Netzdichte, die Elementart und den gewählten Berechnungsalgorithmus.

Das Eigenwertproblem. Bei dieser Analyseform besteht das Ziel in der Ermittlung verschiedener möglicher Lastzustände. Das Verfahren eignet sich sowohl bei stationären als auch bei dynamischen Berechnungen. Meistens stellt sich dabei folgende Frage (vgl. [2]): Gibt es neben der stationären Lösung des Systems eine weitere Lösung, in die das System übergehen kann, wenn es in seiner Gleichgewichtslage geringfügig gestört wird?

Bei Knickstäben handelt es sich um ein Eigenwertproblem, da sich das Bauteil unter Druck zuerst ausbeult bevor es sich deformiert. Eigenwertprobleme sind häufig bei Stab- und Blechkonstruktionen anzutreffen. Knickprobleme unterteilen sich in Knicken erster und zweiter Ordnung (vgl. [2]). In dieser Arbeit betrachten wir das Knicken erster Ordnung.

Der Elementtyp. Zur Modellierung der Objekte stehen drei verschiedene Elementgruppen zur Verfügung. Deren Einsatz ist dem entsprechenden Objekt und Problem anzupassen. Dabei wird zwischen Stab-, Flächen- und Volumenelementen unterschieden. Der Berechnungsaufwand steigt mit zunehmender räumlicher Dimension. Innerhalb dieser Elementgruppen sind verschiedene Elementtypen angesiedelt, die sich durch Form und mathematische Ordnung unterscheiden. Elemente erster Ordnung besitzen in jedem Eckpunkt einen Berechnungsknoten. Elemente zweiter Ordnung haben zusätzliche Knoten zwischen den Eckknoten. Es gilt der Grundsatz: Je mehr Knoten ein Element besitzt, desto flexibler verhält es sich in der Berechnung. Lineare Elemente besitzen somit die Tendenz, Bauteile 
zu versteifen und somit Spannungs- und Deformationsverläufe ungenügend abzubilden. Sie werden in der Industrie jedoch häufig für schnelle, grobe Abschätzungen eingesetzt.

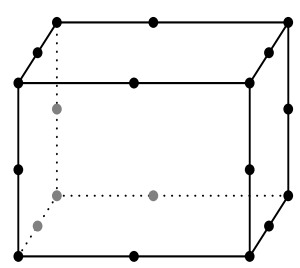

Abb. 9 Brickelement, aussen.

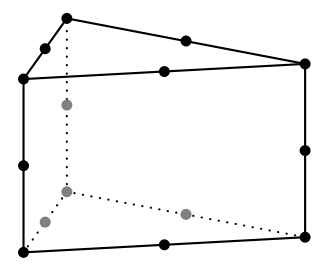

Abb. 10 Prismaelement, innen

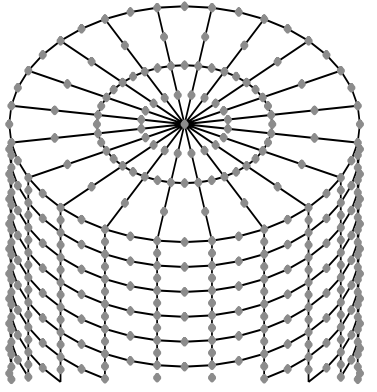

Abb. 11 FE-Netz am Stabende.

Der gesamte Stab setzt sich aus einer Elementschale und einem Elementzylinder zusammen (vgl. Abb. 11). Der äussere Ring besteht aus Brickelementen (vgl. Abb. 9) und der innere Zylinder aus Prismen (vgl. Abb. 10).

Die Randbedingungen. Die Kraft setzt auf der Staboberseite am zentrischen Knoten an (vgl. Abb. 11). Die Festhaltungen sind jeweils an den zentrischen Knoten an der Ober- und Unterseite des Stabes angebracht. Die Translationen an der Oberseite des Stabes sind in $x$ und $y$-Richtung und an der Unterseite in $x$-, $y$ - und $z$-Richtung festgehalten; zudem ist die $z$-Rotation festgehalten. So entsteht beidseitig ein gelenkiges Lager, das die Rotationen in der $x y$-Ebene überträgt.

Die Netzvalidierung. Die Genauigkeit der Lösung bei FEM-Untersuchungen hängt sehr stark vom einbeschriebenen Netz ab. Durch das Netz wird die Geometrie des Objekts angenähert. Die Schwierigkeit besteht darin, ein möglichst grobes Netz in das Modell zu legen, das dennoch den Qualitätsanforderungen entspricht. Bei kritischen Problemen sind mehrere Berechnungen mit unterschiedlichen Netzdichten durchzuführen. Dabei ändert sich ab einer gewissen Netzdichte das Resultat auch bei zunehmend feinerer Vernetzung nicht mehr.

Die Berechnungsmodelle. Wir betrachten fünf Stabtypen: Doppelkonus verjüngt, Konus, Zylinder, Doppelkonus verdickt und technisch optimaler Stab (vgl. Abb. 12). Alle Stäbe besitzen jeweils dasselbe topologische Netz. Zur Vernetzung verwenden wir ein rotationssymmetrisches Netz mit zwei Unterteilungen senkrecht zum Radius, 20 Unterteilungen in radialer und 450 Unterteilungen in axialer Richtung. Total entspricht dies 18000 Elementen.

Den optimalen Stab betrachten wir genauer: Das Netz in axialer Richtung widerspiegelt exakt die analytisch berechnete Geometrie für den optimalen Stab. Diese setzt sich aus konischen Kegelstümpfen mit einer Höhe von einem Millimeter zusammen. Diese feine Unterteilung wäre eigentlich nicht nötig, sie ist aber zur besseren Vergleichbarkeit zwischen der analytischen und der finiten Elemente Berechnung entsprechend der Schrittweite des numerischen Modells übernommen worden. Die Lösung wird iterativ angenähert. 
Aus diesem Grund variieren die Rechenzeiten der einzelnen Stäbe. Die Simulationen wurden auf einer Workstation mit Hilfe der Software ADINA (vgl. [1]) berechnet. Die daraus resultierenden Knicklasten sind in Tab. 1 zum Vergleich mit der analytischen Rechnung und dem Experiment aufgeführt.

\section{Experimente}

Die Wahl des Werkstoffes. Entscheidend für die Wahl des Werkstoffes waren die Bearbeitbarkeit - es galt Probleme bei der Herstellung zu vermeiden - und die Grösse des Elastizitätsmoduls, da dieser direkt linear die Knicklast beeinflusst (vgl. Kap. 2). Den Berechnungen liegt die Annahme einer reibungsfreien, gelenkigen Lagerung zu Grunde, demzufolge kann die Einspannung kein Moment übernehmen. Um dies in den Versuchen möglichst zu realisieren, müssen die Kräfte auf die Gelenklager klein gehalten werden, da damit auch die Reibung minimal ausfällt. Die gewählte Aluminium-Legierung (Anticorodal-112, warm ausgehärtet T6, EN AW-6082 AlSi1MgMn) bietet eine optimale Lösung, ihre Eigenschaften erfüllen alle Anforderungen. Mit diesem in der Technik oft verwendeten Werkstoff ergeben sich Knicklasten, die gegenüber Stahl um den Faktor drei kleiner sind.

Die Herstellung der Knickstäbe. Die Fertigung der Stäbe erfolgte aus einem Halbzeug, dabei wurden die Rohlinge aus einem stranggepressten Rundstab mit einem Durchmesser von $25 \mathrm{~mm}$ zugeschnitten. Anschliessend folgte die spanende Bearbeitung auf einer CNC-Drehmaschine mit zwei synchron laufenden Spindeln. Dies erlaubte ein beidseitiges Einspannen der Stabenden, was die Steifigkeit erhöhte. Damit gelang es Knickstäbe zu produzieren, die eine grosse Formtreue, sehr gerade Achsen und eine optimale Oberflächenbeschaffenheit aufweisen. Für die Experimente wurden Stäbe mit den in Abb. 12 gezeigten Geometrien hergestellt. Damit besitzen alle Stäbe dasselbe theoretische Volumen von $114511 \mathrm{~mm}^{3}$ resp. die gleiche theoretische Masse von $309.2 \mathrm{~g}$, und ihre Länge ist identisch, was für den direkten Vergleich erforderlich ist.

Die Zugversuche zur Ermittelung der exakten Werkstoffkennwerte. Da die Angaben von Rohmaterial-Lieferanten bezüglich der Werkstoffeigenschaften immer nur Richtoder Mindestwerte darstellen, wurde es vorgezogen, die effektiven Kennwerte mittels eigenen Zugversuchen selbst zu ermitteln. Aus vier Norm-Zugproben ergaben die folgenden Mittelwerte der mechanischen Werkstoffkennwerte:

$$
\begin{array}{ll}
\text { Elastizitätsmodul } & E=71290 \mathrm{~N} / \mathrm{mm}^{2}, \\
\text { Streckgrenze (Rp0.2) } & \sigma_{0.2}=372 \mathrm{~N} / \mathrm{mm}^{2}, \\
\text { Zugfestigkeit (Rm) } & \sigma_{\mathrm{Z}}=390 \mathrm{~N} / \mathrm{mm}^{2}, \\
\text { Bruchdehnung (A5) } & \varepsilon_{\text {Bruch }}=10.4 \% .
\end{array}
$$

Im Spannungs-Dehnungs-Diagramm (vgl. Abb. 13) ist der Elastizitätsmodul als Steigung der Hookeschen Gerade zu erkennen. In der Auswertung jedes Zugversuches wurde er durch eine lineare Regression der Spannung im Bereich von 30 bis $260 \mathrm{~N} / \mathrm{mm}^{2}$ ermittelt.

Die Einspannung der Stäbe, die Zugmaschine und das Gelenklager. Allen Berechnungen in diesem Artikel liegt der zweite Eulersche Fall mit gelenkig gelagerten Stabenden zu Grunde. Die Einspannstellen sind somit reibungsfreie Gelenke, die kein Moment 

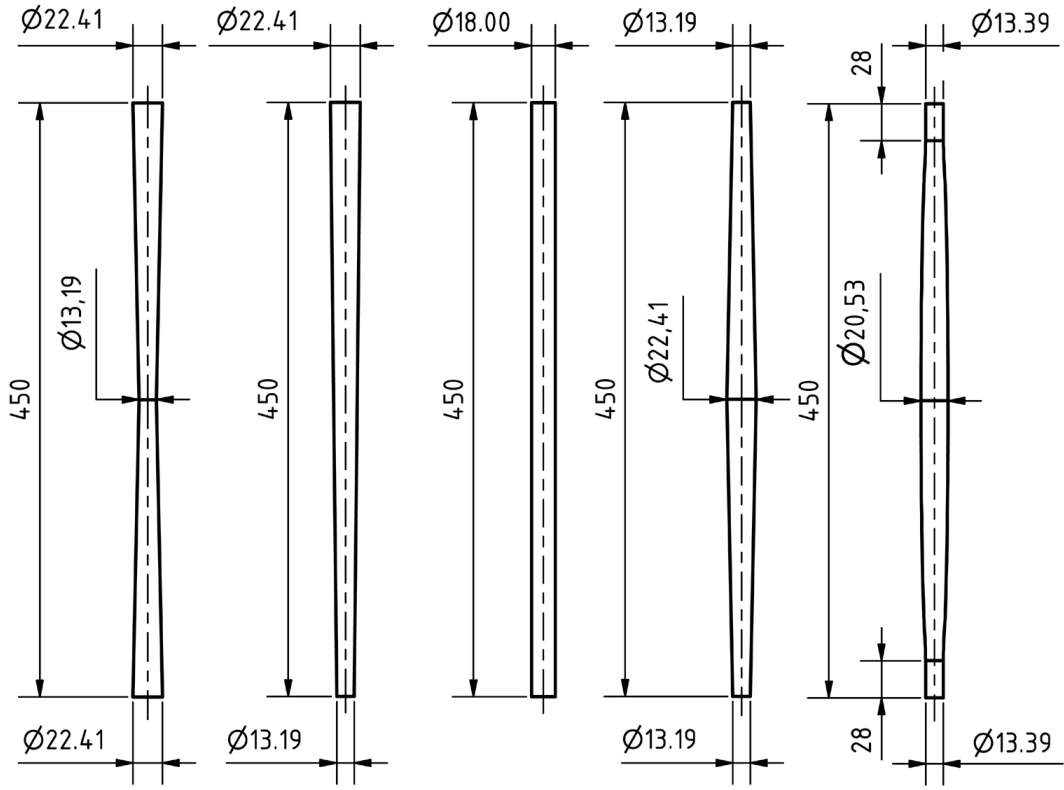

Abb. 12 Geometrien der Knickstäbe: Doppelkonus verjüngt, Konus, Zylinder, Doppelkonus verdickt und technisch optimaler Stab (v.1.n.r.); Angaben in mm.

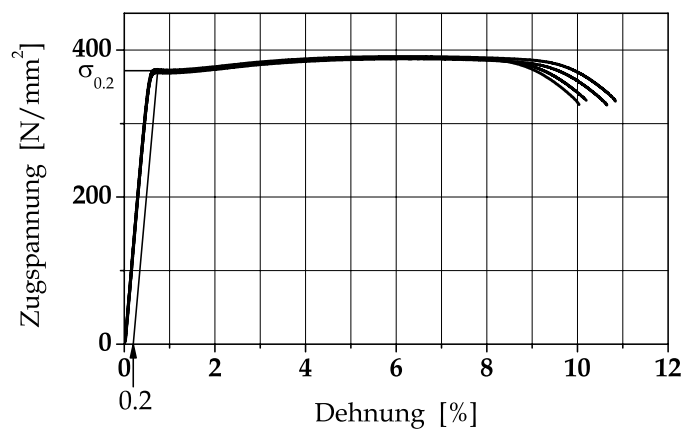

Abb. 13 Spannungs-Dehnungs-Diagramm der vier Zugproben.

aufnehmen können. Es gibt zwei wichtige Gründe, weshalb gerade diese Einspannart untersucht wurde. Zum einen, weil damit die Knicklasten am kleinsten ausfallen und zum anderen, da diese technisch am einfachsten zu realisieren ist. Alle Knickversuche und auch die Bestimmung der Werkstoffkennwerte wurden auf einer Zugmaschine vom Typ Zwick ZL 100 durchgeführt. Die Knickversuche erforderten jedoch spezielle Vorrichtungen für die Positionierung der Stabenden. Diese sogenannten Gelenklager wurden eigens dazu hergestellt (vgl. Abb. 14 und 15). Dabei gewährte der Einsatz spezieller Kugellager eine minimale Reibung. Weitere konstruktive Ausführungen sorgten für eine gute Übereinstimmung zwischen mathematischem und experimentellem Modell. 


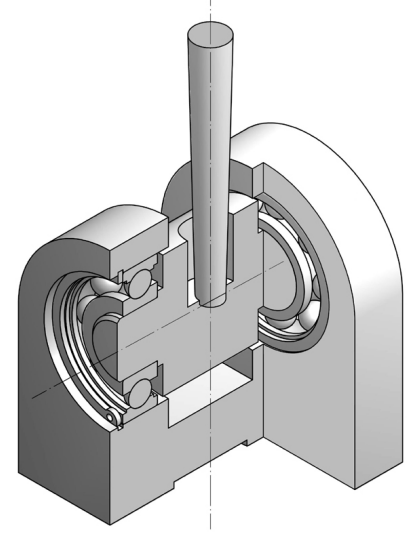

Abb. 14 Schnittzeichnung des Gelenklagers.

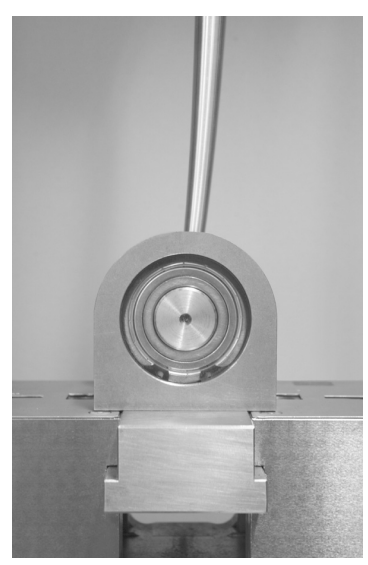

Abb. 15 Gelenklager mit Knickstab unter Last.

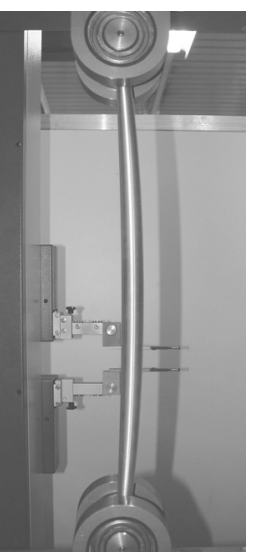

Abb. 16 Knickstab in Zugmaschine unter Last.

Die Ausführung der Knickversuche. Für die Knickversuche wurden die Stäbe zwischen zwei Gelenklagern eingespannt. Dazu wurde ein Lager oben am ruhenden Joch der Prüfmaschine befestigt und das andere auf der verschiebbaren Traverse (vgl. Abb. 16). Das Hochfahren der Traverse staucht die Stäbe, sie erfahren dadurch eine axiale Druckbelastung. Die Knickversuche erfolgten unter Regelung des Fahrweges, womit die Traverse stets mit einer konstanten Geschwindigkeit gefahren wurde. Dies ermöglichte eine genaue Untersuchung des Knickvorganges, da der Kraftanstieg stets kontinuierlich erfolgte. Bei allen untersuchten Knickstäben liegt die maximale Biegespannung beim Ausknicken unterhalb der Streckgrenze des verwendeten Stabwerkstoffes. Das Knicken findet demzufolge eindeutig im elastischen Bereich des Materials statt. Demnach handelt es sich hier um ein Stabilitäts- und nicht um ein Festigkeitsproblem. Der Vorgang des Ausknickens ist in Abb. 17 auf verschiedene Weise dargestellt.
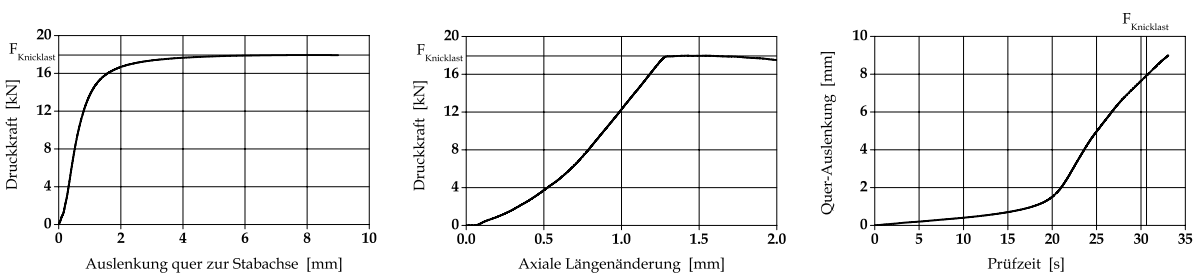

Abb. 17 Belastungsdiagramme des zylindrischen Stabes mit der Druckkraft in Funktion der radialen und axialen Auslenkung, sowie der Querauslenkung in Funktion der Zeit.

Da die seitliche Auslenkung zuerst langsam und dann kurz vor Erreichen der maximalen Last plötzlich sehr rasch erfolgt, ist die exakte Bestimmung der Knicklast mit Unsicherheiten verbunden. Bei der Auswertung der Messungen wurde die Knicklast durch das Kraftmaximum festgelegt, welches diese gemäss den Beobachtungen mit grosser Genauigkeit repräsentiert. Wenn vor Erreichen der maximalen Kraft eine Entlastung des ausgelenkten 
Stabes erfolgt, kehrt dieser sofort wieder in seine Ausgangslage zurück. Dabei bleibt seine Achse gerade und er zeigt keine Anzeichen einer plastischen Deformation.

Der Verlauf der Biegespannung. Es bezeichnen $d(x)$ den Durchmesser und $y(x)$ die Biegelinie in Funktion des Abstandes $x$ auf der Stabachse. Dann ist die Biegespannung durch

$$
\sigma_{\mathrm{B}}(x)=\frac{1}{2} y^{\prime \prime}(x) d(x) E
$$

gegeben. Der genaue Wert der Biegespannung lässt sich jedoch nur ermitteln, wenn die Auslenkung entlang der Stabachse bekannt ist. Die Berechnung der Biegelinie beinhaltet immer einen unbekannten, konstanten Amplitudenfaktor (vgl. Kap. 2). Dieser könnte experimentell, durch Messen der Auslenkung an einer definierten Stelle des Stabes, bestimmt werden. Davon wurde aber in den Versuchen abgesehen. Der Verlauf der Biegespannung kann jedoch ohne grossen Aufwand ermittelt werden, er ist für die verschiedenen Stabgeometrien in Abb. 18 dargestellt.
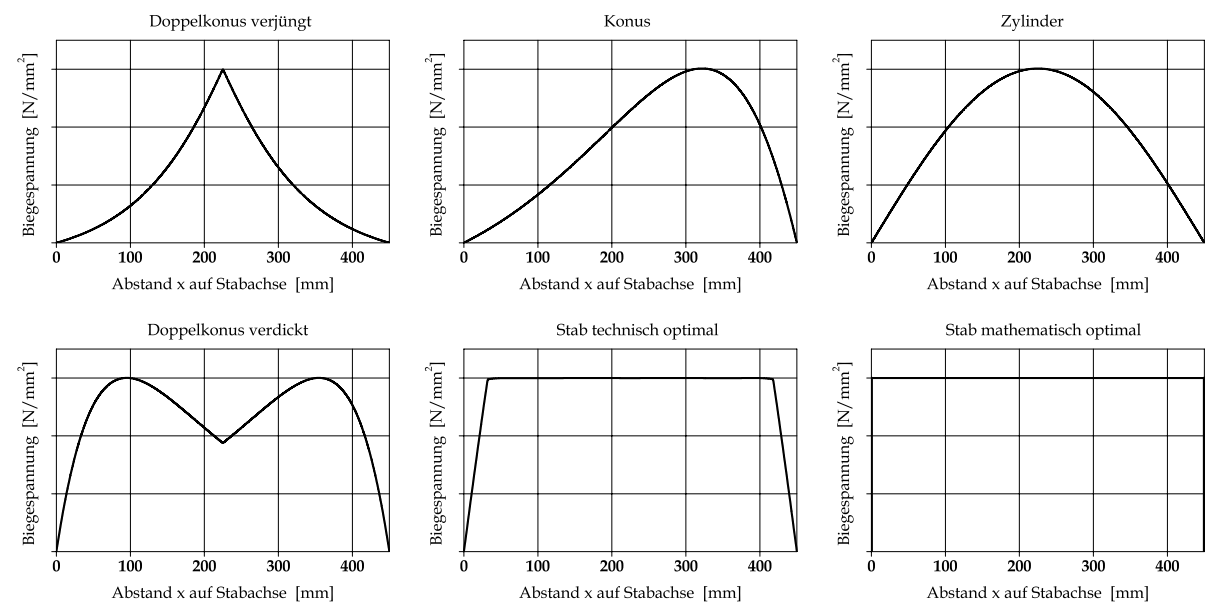

Abb. 18 Verlauf der Biegespannungen der Knickstäbe. Die exakten Amplituden sind unbekannt.

Der optimale Knickstab zeichnet sich dadurch aus, dass die Biegespannung entlang seiner Achse an konstant ist. Damit ist er auf der gesamten Länge gleich beansprucht, und die Festigkeit des Werkstoffes kann voll ausgenutzt werden. Dies wird jedoch nur vom mathematisch optimalen Stab erfüllt, der aber technisch nicht sinnvoll ist (vgl. Gl. (10)).

\section{Resultate und Diskussion}

In Tab. 1 sind die einzelnen Knicklasten, die mit den verschiedenen Methoden ermittelt wurden, aufgeführt. Für die Experimente wurden pro Stabtyp zwei Exemplare hergestellt und zur Kontrolle der Fertigungsqualität deren Massen bestimmt. Mit den beiden Exemplaren wurden dann die Knicklasten auf der Zugmaschine bestimmt. Die FEM-Rechnungen und die analytischen Rechnungen lieferten je Stabtyp genau eine Knicklast. 


\begin{tabular}{l||r||r|r|r}
\multicolumn{1}{l||}{ Stabtyp } & \multicolumn{1}{c||}{ Stabmasse } & \multicolumn{3}{c}{ Knicklasten } \\
& & Experiment & $\begin{array}{r}\text { FEM- } \\
\text { Rechnung }\end{array}$ & $\begin{array}{c}\text { analytische } \\
\text { Rechnung }\end{array}$ \\
\hline \hline $\begin{array}{l}\text { Doppelkonus } \\
\text { verjüngt }\end{array}$ & $\begin{array}{r}310.2 \mathrm{~g} \\
309.4 \mathrm{~g}\end{array}$ & $9446 \mathrm{~N}$ & $9517 \mathrm{~N}$ & $9500 \mathrm{~N}$ \\
\hline Konus & $311.1 \mathrm{~g}$ & $14931 \mathrm{~N}$ & $14925 \mathrm{~N}$ & $14911 \mathrm{~N}$ \\
& $310.9 \mathrm{~g}$ & $14868 \mathrm{~N}$ & & \\
\hline Zylinder & $309.1 \mathrm{~g}$ & $17938 \mathrm{~N}$ & $17922 \mathrm{~N}$ & $17905 \mathrm{~N}$ \\
& $310.9 \mathrm{~g}$ & $18022 \mathrm{~N}$ & & \\
\hline Doppelkonus & $310.7 \mathrm{~g}$ & $22557 \mathrm{~N}$ & $22274 \mathrm{~N}$ & $22277 \mathrm{~N}$ \\
verdickt & $314.2 \mathrm{~g}$ & $22528 \mathrm{~N}$ & & \\
\hline Stab technisch & $309.4 \mathrm{~g}$ & $23078 \mathrm{~N}$ & $22882 \mathrm{~N}$ & $22945 \mathrm{~N}$ \\
optimal & $309.9 \mathrm{~g}$ & $23048 \mathrm{~N}$ & & \\
& & &
\end{tabular}

Tab. 1 Experimentell bestimmte und berechnete Knicklasten $F_{\text {Knicklast }}$ der einzelnen Stäbe.

Diskussion der Resultate. Die Mittelwerte der experimentell ermittelten Knicklasten weichen nur wenig von den analytisch resultierenden Werten ab. Die Differenzen liegen im Bereich von $-1.2 \%$ bis $+1.2 \%$ und sind damit auffallend klein. Erwartungsgemäss gering hingegen sind die Unterschiede von $-0.2 \%$ bis $+0.3 \%$ zwischen den FEM- und den analytischen Ergebnissen.

Die nach der Ermittlung der Werkstoffkennwerte ausgeführten Knickversuche zeigten, dass das Ausknicken der Stäbe deutlich am oberen Ende des elastischen Bereiches erfolgt. Wenn nun dieser Umstand bei der Bestimmung des Elastizitätsmoduls durch Heraufsetzen der oberen Regressionsgrenze auf 300 bis $340 \mathrm{~N} / \mathrm{mm}^{2}$ berücksichtigt wird, resultieren daraus durchwegs kleinere Elastizitätsmodule. Diese liegen in der Grösse von 69568 bis $70711 \mathrm{~N} / \mathrm{mm}^{2}$, was einer Reduktion von 0.8 bis $2.4 \%$ gegenüber dem verwendeten Wert entspricht. Da der Elastizitätsmodul direkt proportional in die Berechnung der Knicklast eingeht (vgl. Kap. 2), müssten die rechnerisch ermittelten Knicklasten damit auch in dieser Grössenordnung kleiner ausfallen.

Die verdickten Doppelkonusse wurden nachträglich auf einer konventionellen Drehmaschine angefertigt. Deshalb ist die Streuung ihrer Massen grösser als bei allen anderen Stäben, die mit der Zweispindel CNC-Drehmaschine hergestellt wurden. Eventuell stammen die Rohlinge dieser Stäbe auch aus einer anderen Werkstoffcharge mit einem leicht grösseren Elastizitätsmodul, was wiederum eine leicht erhöhte Knicklast zur Folge hätte und damit den grössten Unterschied bei den Resultaten erklären würde (vgl. Tab. 1).

Danksagung. Die Autoren danken dem Institut für Produkt- und Produktionsengineering (IPPE) der FHNW für die Bereitstellung ihrer Laboreinrichtungen, die Fertigung der Stäbe und für die zur Verfügung gestellte Zeit. Wir danken René Kaiser und Kevin Martin vom IPPE für nachträgliche FEM-Simulationen. 


\section{Literatur}

[1] ADINA 2006, ADINA-AUI Documentation.

[2] Bathe, K.J.: Finite-Elemente-Methode. Springer Verlag, 2002.

[3] Katsikadelis, J.T.; Tsiatas, G.C.: Buckling load optimization of beams. Arch. Appl. Mech. 74 (2005), 790799.

[4] Keller, J.B.: The shape of the strongest column. Arch. Rational Mech. Anal. 5 (1960), 275-285.

[5] Maalawi, K.Y.: Buckling optimization of flexible columns. Internat. J. Solids Structures 39 (2002), 58655876.

[6] Meschede, D.; Gerthsen, C.: Gerthsen Physik. Springer Lehrbuch, 21. Auflage, 2001.

[7] Nelder, J.A.; Mead, R.: A simplex algorithm for function minimization. The Computer Journal 7 (1965), 308-313.

[8] R Development Core Team: R: A language and environment for statistical computing (2009), www. R-project.org.

[9] Szabó, I.: Geschichte der mechanischen Prinzipien. Birkhäuser, 1977.

[10] Timoshenko, S.P.: History of strength of materials. Dover Publications, 1983.

[11] Truesdell, C.: The rational mechanics of flexible or elastic bodies 1638-1788. In: L. Euleri Opera Omnia Series II, vol. 11, part 2, Zürich 1960.

Philippe Cachot

Helbling Technik AG

Schachenallee 29

CH-5000 Aarau, Schweiz

e-mail: philippe.cachot@helbling.ch

Markus Meier

Alte Kantonsschule Aarau

Bahnhofstrasse 91

CH-5000 Aarau, Schweiz

e-mail: markus.meier@alte-kanti-aarau.ch

Marcel Steiner

Hochschule für Technik FHNW

Steinackerstrasse 5

CH-5210 Windisch, Schweiz

e-mail: marcel.steiner@fhnw.ch

Urs Wüst

array-spotting

Burgfelderstrasse 26, P.O. Box 377

CH-4012 Basel, Schweiz

e-mail: info@array-spotting.com 